\title{
Non-uniform sound intensity distributions when measuring absorption coefficients in reverberation chambers using a phased beam tracing
}

Jeong, Cheol-Ho

Published in:

Acoustical Society of America. Journal

Link to article, DOI:

$10.1121 / 1.3397475$

Publication date:

2010

Document Version

Publisher's PDF, also known as Version of record

Link back to DTU Orbit

Citation (APA):

Jeong, C-H. (2010). Non-uniform sound intensity distributions when measuring absorption coefficients in reverberation chambers using a phased beam tracing. Acoustical Society of America. Journal, 127(6), 35603568. https://doi.org/10.1121/1.3397475

\section{General rights}

Copyright and moral rights for the publications made accessible in the public portal are retained by the authors and/or other copyright owners and it is a condition of accessing publications that users recognise and abide by the legal requirements associated with these rights.

- Users may download and print one copy of any publication from the public portal for the purpose of private study or research.

- You may not further distribute the material or use it for any profit-making activity or commercial gain

- You may freely distribute the URL identifying the publication in the public portal 


\title{
Non-uniform sound intensity distributions when measuring absorption coefficients in reverberation chambers using a phased beam tracing ${ }^{\text {a) }}$
}

\author{
Cheol-Ho Jeong ${ }^{\text {b) }}$ \\ Department of Electrical Engineering, Acoustic Technology, Technical University of Denmark, \\ Kongens Lyngby DK-2800, Denmark
}

(Received 11 February 2009; revised 23 March 2010; accepted 23 March 2010)

\begin{abstract}
Measured absorption coefficients in reverberation chambers often differ from theoretical random incidence absorption coefficients, because ideal assumptions for the theoretical random incidence absorption coefficient are not fulfilled during measurements in actual reverberation chambers. Therefore sound intensity distributions on absorber under measurement conditions have been simulated using a phased beam tracing, and used as correction functions for reducing discrepancies between the measured and theoretical absorption coefficients. Two reverberation rooms were investigated by assuming that a test specimen was attached to a vertical surface and the floor. The frequency-dependent sound intensity distributions on absorbers were found to be affected by the reverberation chamber geometry and dimensions, the absorption capability of the specimen, and the placement of the specimen. High frequency intensity distributions above $1 \mathrm{kHz}$ were similar for all studied cases, but some variations in low frequency intensity distributions were observed. If the non-uniform intensity distribution and a finite size effect are taken into account for correcting the theoretical absorption coefficients, a good agreement is found between corrected and measured statistical absorption coefficients. The non-uniform sound intensity can account for the discrepancy at high frequencies. (C) 2010 Acoustical Society of America. [DOI: 10.1121/1.3397475]
\end{abstract}

PACS number(s): 43.55.Ev, 43.55.Nd [LMW]

Pages: $3560-3568$

\section{INTRODUCTION}

It is well known that statistical absorption coefficients measured in reverberation chambers differ from theoretical random incidence absorption coefficients. ${ }^{1}$ The theoretical random incidence absorption coefficient for plane wave incidence and locally reacting surfaces can be calculated solely from the specific surface impedance as follows: ${ }^{2}$

$$
\begin{aligned}
\alpha_{\text {rand }}= & \frac{8}{|\zeta|^{2}} \cos \beta\left[|\zeta|+\frac{\cos 2 \beta}{\sin \beta} \arctan \left(\frac{|\zeta| \sin \beta}{1+|\zeta| \cos \beta}\right)\right. \\
& \left.-\cos \beta \ln \left(1+2|\zeta| \cos \beta+|\zeta|^{2}\right)\right]
\end{aligned}
$$

where $\zeta$ means the specific surface impedance and $\beta$ is the phase angle of the specific surface impedance. It assumes that the intensity of the incident sound is uniformly distributed over all possible directions, and the phases of the waves incident on the absorber are randomly distributed. In addition, the absorber under test is assumed to be infinitely large. However, the statistical absorption coefficients measured by the reverberation chamber method do not perfectly fulfill the underlying assumptions of the theoretical random incidence absorption coefficient. First, reverberation rooms are not completely diffuse, meaning that some angles of incidence are emphasized over others. Second, test specimens are finite, which leads to an overestimation of the measured ab-

\footnotetext{
Portions of this study were presented in "Sound intensity on an absorber in reverberant conditions," Proceedings of the Inter-noise 2009, Ottawa, Canada, August 2009.

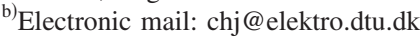

sorption coefficients at low frequencies due to diffraction invoked by the free edges of the test specimens ${ }^{3,4}$ and a size effect. ${ }^{5}$ Other reasons for the discrepancies are the finite volume of a reverberation chamber, ${ }^{6}$ the locations of sample/source/microphones, ${ }^{7-9}$ the mounting methods, ${ }^{9}$ the geometry and size of an absorber, ${ }^{10}$ the use of the Sabine formula, ${ }^{11,12}$ and the non-random phases of the incident wave components. This study focuses mostly on the non-diffuse sound field, and in particular, the non-uniform sound intensity on absorbers in reverberation chambers using a numerical phased beam approach, and it is found that this information can be used to alleviate the discrepancies between the theoretical random incidence and the statistical absorption coefficients significantly.

Measured absorption coefficients in reverberation chambers are estimated from the decay of sound under measurement conditions. The overall sound decay in a room consists of a number of normal modes of vibration, each having its own attenuation characteristic depending on its orientation with respect to the absorbing wall and the normal impedance of the absorbing material. ${ }^{13}$ In the literature one can find several modal approaches to account for the discrepancies between measured and theoretical absorption coefficients. Hunt and co-workers ${ }^{14}$ divided all the normal modes in a frequency band into subgroups of excited modes and applied a weighting factor to each subgroup of normal modes. The main principle of grouping room modes is that the grazing incidence on an absorber sample has different significances from non-grazing incidence components. Three weighting factors of 1,1/2, and 1/4 were adopted for oblique, tangential, and axial modes, respectively, and by summing all the 
weighted decays, the overall decay rate was obtained and compared with measurements. Assuming that the shortest dimension of the room is the height and that an absorber is mounted on the floor, there exist an excessive number of grazing modes on the absorber. ${ }^{15}$ This may lead to the conclusion that a more uniform angular distribution of incident energy on the absorber would be obtained by mounting the test specimen perpendicular to the longest dimension rather than on the floor. Bolt also applied an angular modal distribution to the calculation of average absorption coefficient. ${ }^{16}$ Such wave based approaches are mainly useful for low frequency corrections, where the tangential and axial modes are fairly dominant. As the frequency increases, the modal effect becomes less significant. Moreover, these approaches are only restricted to the sound decay in rectangular rooms containing no sound scattering objects, with uniform and perfectly reflective walls. In principle, the same theory can be also applied to nonrectangular rooms, but the analysis is quite difficult, and in fact, almost impossible for complicated room geometries.

Yet another possible approach is to use a geometrical acoustics model mainly for determining high frequency corrections. The introduction of a highly absorbing patch in a reverberant room, which definitely influences the sound field and the modal distribution, can be taken into consideration in the geometrical acoustics models. Such methods can also deal with complex room geometries with non-uniform absorption distributions. Using the geometrical acoustics models, the actual incident energy distribution in a room can be estimated and used as a correction factor. ${ }^{17}$ Phased geometrical acoustics techniques, ${ }^{18}$ which retain phase information during ray/beam tracing, can also be used in such analyses. A phased beam tracing technique has been used for calculating sound intensities in this study, and a brief introduction to the phased beam tracing method is given in Sec. II A.

There have been attempts to elucidate the discrepancies between theoretical acoustic quantities and measured ones in reverberation chambers on the basis of the non-uniform energy distribution. The field incidence mass law, which is obtained by integrating an angle dependent transmission coefficient from $0^{\circ}$ to $78^{\circ}$, indicates non-uniform sound incidence onto test specimens in practical conditions. ${ }^{19}$ Furthermore, better agreement was found using smoothly varying angular distributions of incident energy rather than using a sharp truncation at $78^{\circ}$ by Kang and co-workers. ${ }^{20}$ Makita and Hidaka $^{21}$ discussed possible variations of absorption coefficients in non-uniform energy field. Recently, Jeong ${ }^{17}$ investigated incident energy density distributions for a rectangular and an irregular room using a beam tracing technique. The simulated incident energy density distributions as a function of the incidence angle for different source locations were used as weighing functions for correcting theoretical absorption coefficients. Out of several weighting functions, a relatively flat weighting produces better agreement at low frequencies, whereas a gradually decreasing Gaussian-like distribution yields more accurate results at high frequencies and for large samples in comparison with measured data. The main drawback of the incident energy density is its frequency-independent characteristic, which is the result of the assumption that all surfaces are quite reflective regardless of the frequency. Moreover, only spatially averaged incident energy density distributions over the surface of interest is predicted. However, it is often of interest to observe local phenomena; for example, to see how the sound intensity differs from one position near the central part to another near a corner of the test specimen. Therefore sound intensity distributions at some positions on absorbers installed in two reverberation chambers were investigated, and used as weighting functions to reduce the discrepancies between the measured and the theoretical absorption coefficients.

\section{METHOD}

\section{A. Sound pressure calculation using the phased beam tracing method}

The phased beam tracing technique has been used to investigate the intensity distributions on the absorbers. The applicability of the phased method can be extended to the low and mid frequency ranges by taking account of interference of waves. Phase shifts on reflections are taken into account by use of complex-valued pressure reflection coefficients. As a result, "sound pressures" at specified receivers are obtained by the phased beam tracing methods, whereas "energy quantities" are calculated by the geometrical acoustics models. The pressure at a receiver location by a beam is computed based on information of the travel distance and surfaces that the beam hits, given by

$$
p\left(t=\frac{d_{\mathrm{tot}}}{c_{o}} ; \theta_{i}, \omega\right)=\frac{p_{s}}{d_{\mathrm{tot}}} e^{-j \hat{k} d_{E}} \prod_{i=1}^{q} r_{i}\left(\theta_{i}, \omega\right)
$$

where $p_{s}$ is the initial pressure amplitude at the source, $d_{\text {tot }}$ is the total travel distance of the beam, $\tilde{k}$ is the complex wave number, $\widetilde{k}=k-j 0.5 m, k$ is the wave number in a lossless free field, $m$ is the air attenuation factor, $r_{i}\left(\theta_{i}\right)$ is the pressure reflection coefficient of the $i$ th wall reflection, $\theta_{i}$ is the angle of incidence of the beam to the $i$ th wall, $q$ is the total number of wall reflections until the beam reaches the receiver, $c_{o}$ is the speed of sound in air, and $\omega$ is the angular frequency. It is noted that the pressure calculated by Eq. (2) is complexvalued for a single frequency and for a single beam.

A proper use of the pressure reflection coefficient of a surface is of utmost importance in the phased beam tracing method. Commonly the walls of reverberation chambers are quite reflective, so the absorption coefficient of the bare walls $\alpha_{w}$ is assumed to be 0.03 . A real pressure reflection coefficient $r_{w}=\sqrt{1-\alpha_{w}}=0.985$ can be assumed, because phase shifts are negligible for acoustically hard walls.

However, since absorbers are not reflective, two pressure reflection characteristics were employed. One is a real pressure reflection coefficient computed in the same way with the bare walls, given by $r_{\text {real }}=\sqrt{1-\alpha_{s}}$, in which the subscript $s$ refers to the absorber specimen. However, neglecting the phase shift from the absorber sample can cause some errors, in particular, for highly absorbing materials and grazing incidence cases. Therefore a complex pressure reflection coefficient obtained from surface impedance data was also 

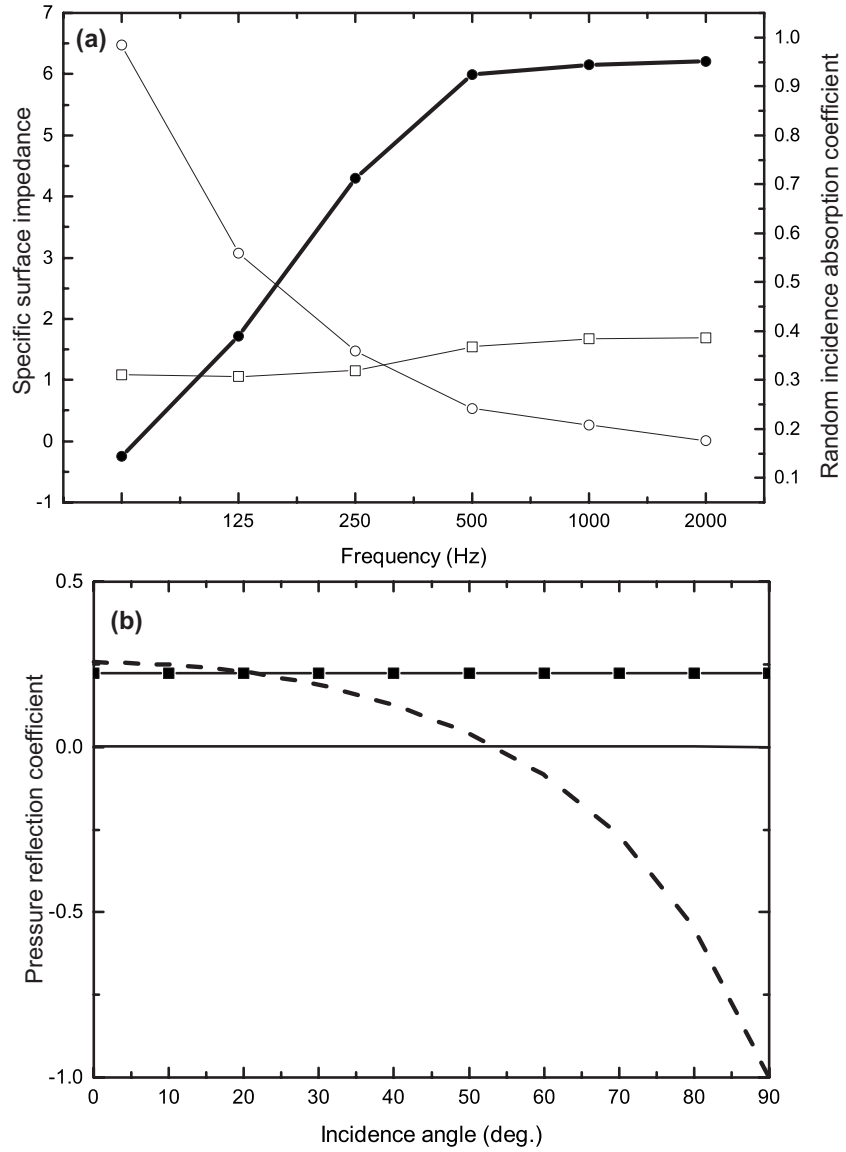

FIG. 1. Acoustical properties of a test material; (a) specific surface impedance and absorption coefficient and (b) pressure reflection coefficients: ——: $\operatorname{Re}(\zeta) ;-\bigcirc —: \operatorname{Im}(\zeta) ;-\bigcirc —: \alpha_{\text {rand }} ;-\square —: r_{\text {real }} ;$ and ---: $\operatorname{Re}\left(r_{\text {comp }}\right)$.

used in the simulations. The complex pressure reflection coefficient for plane wave incidence is given by

$$
r_{\mathrm{comp}}=\frac{\zeta \cos \theta-1}{\zeta \cos \theta+1} .
$$

Here, $\theta$ is the incidence angle. Equation (3) basically assumes an infinite and locally reacting panel. The infinite panel assumption may cause errors near grazing incidence and for small surfaces, which is well discussed in Ref. 22. For the material from Ref. 23, the surface impedance data, the random incidence absorption coefficient, and two pressure reflection coefficients are shown in Fig. 1. According to Fig. 1(b), it is apparent that the complex pressure reflection coefficient differs from the real pressure reflection coefficient above the incidence angle of $30^{\circ}$. The real part of the complex pressure reflection coefficient becomes even negative above $50^{\circ}$, which might result in destructive interference in the sound field.

\section{B. Sound intensity calculation}

Sound intensity is the time averaged product of pressure and particle velocity, which can be computed using two scalar sound pressures at two calculation points. ${ }^{24}$ It is a vector quantity, so the direction of net flow of acoustic energies is determined by the locations of the two observation points. The particle velocity at the midpoint between the two calcu- (a)

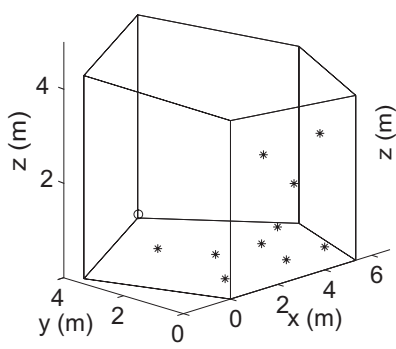

(b)

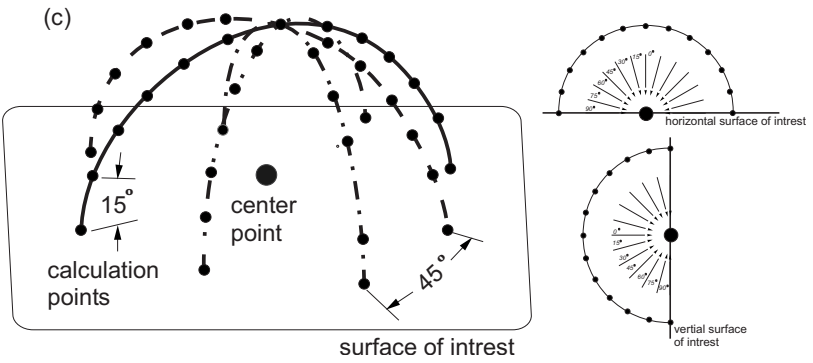

FIG. 2. Room models and receivers: (a) irregular room, (b) rectangular room, and (c) a set of calculation points. "o" denotes the source and "**" denotes the receiver.

lation points is estimated by integrating the pressure gradient, whereas the pressure at the midpoint is computed by averaging the two pressures. From two pressures, $p_{1}$ and $p_{2}$, the sound intensity averaged over the range $[t \sim t+T]$ can be calculated as follows:

$I=\frac{1}{2 \rho d T} \int_{t}^{t+T}\left\{\left[p_{1}\left(t^{\prime}\right)+p_{2}\left(t^{\prime}\right)\right] \int_{-\infty}^{t^{\prime}}\left[p_{1}\left(t^{\prime \prime}\right)-p_{2}\left(t^{\prime \prime}\right)\right] d t^{\prime \prime}\right\} d t^{\prime \prime}$,

where $\rho$ is the air density and $d$ is the distance between two calculation points. This method is called the direct integration method. The cross-spectrum between the pressure and the particle velocity yields the sound intensity spectrum, given by

$$
I(f)=-\frac{\operatorname{Im}\left(G_{p 1 p 2}(f)\right)}{2 \pi \rho f d} .
$$

Here, $G_{p 1 p 2}$ denotes the one-sided cross-spectrum between the two pressures $p_{1}$ and $p_{2}$. A band-limited intensity level is estimated by filtering the calculated intensity spectrum.

\section{Reverberation chambers and surfaces of interest}

An oblique-angled room with non-parallel walls in Fig. 2(a) and a rectangular room in Fig. 2(b) were chosen as test examples. These are common geometries of existing reverberation chambers, and the volumes of the reverberation chambers are 179 and $240 \mathrm{~m}^{3}$, respectively. An irregular geometry is regarded as an effective design, since strong standing waves are prohibited to achieve diffuse sound fields.

Reverberation chambers should be of a certain minimum size in order to have enough number of oblique modes at the lowest frequency band. According to ISO 354, the room volume should be larger than $200 \mathrm{~m}^{2}$ considering the lowest one-third octave band centered at $100 \mathrm{~Hz}$, which means at 
TABLE I. Specific surface impedance data of the rockwool from Ref. 23.

\begin{tabular}{lcccccc}
\hline \hline Center frequency (Hz) & 125 & 250 & 500 & 1000 & 2000 & 4000 \\
Specific surface impedance $(\zeta)$ & 1.08 & 1.06 & 1.15 & 1.54 & 1.68 & 1.69 \\
& $+6.48 j$ & $+3.08 j$ & $+1.47 j$ & $+0.53 j$ & $+0.26 j$ & $+0.01 j$ \\
\hline \hline
\end{tabular}

least 14 oblique room modes should exist in the lowest frequency band. $^{25}$ The lowest valid frequency band of the phased beam tracing simulations is $250 \mathrm{~Hz}$ based on Everest's criterion, ${ }^{26}$ because the Schroeder frequencies of the two chambers when containing the absorber sample tested are 180 and $159 \mathrm{~Hz}$. Since there are more than 700 oblique modes in the lowest octave band centered at $250 \mathrm{~Hz}$, both chambers are considered to be large enough for frequencies above $250 \mathrm{~Hz}$.

Another concern is a high frequency limit of the intensity calculations using two pressures, which is determined by the distance between two calculation points, namely, $d$ in Eqs. (4) and (5). The shorter the distance, the higher the frequency limit. Since typically used microphone spacers of $6 \mathrm{~mm}$ in intensity measurements assure precision up to 10 $\mathrm{kHz}$, a distance of $5 \mathrm{~mm}$ was chosen. Therefore the valid frequency range of the prediction is restricted to the interval from $250 \mathrm{~Hz}$ to $4 \mathrm{kHz} 1 / 1$ octave bands.

Two surfaces were examined for the irregular chamber: one is a vertical surface, i.e., the $(x, 0, z)$ plane, and the other is the floor, i.e., the $(x, y, 0)$ plane (coordinates in $\mathrm{m})$. The sound source was located at $(3.72,6,0.1)$ near a corner. The five receiver points on the vertical sidewall are $(2.7,0.001$, $2.5),(1.2,0.001,0.7),(4.0,0.001,0.6),(3.8,0.001,3.6)$, and $(1.4,0.001,3.5)$. For the absorber on the floor, sound intensities have been investigated for five locations, $(2.0,4.0$, $0.001),(2.8,2.7,0.001),(0.7,2.8,0.001),(4.5,2.5,0.001)$, and $(3.9,1.2,0.001)$.

For the rectangular room, only the floor was investigated. Sound intensity distributions were simulated at randomly distributed locations, $(2.7,4.8,0.001)$, (3.4, 4.3, $0.001),(1.5,2.5,0.001),(1.4,3.8,0.001),(3.3,3.1,0.001)$, $(4.3,5.6,0.001),(4.9,3.7,0.001)$, and $(1.7,4.6,0.001)$ for an omni-directional source at $(0.1,0.1,0.1)$. All source (o) and receiver $(*)$ locations are displayed in Figs. 2(a) and 2(b).

The calculated quantity in the present study is net intensity using two sound pressures near the absorber surfaces. However, since the absorbers under test are quite absorptive mostly at high frequencies, the calculated net intensity can be regarded as the incident intensity in an approximate manner. Using such approximate incident intensities from many different directions, an incident intensity distribution can be constructed at a receiver point. Figure 2(c) shows a set of pre-defined 53 calculation points, consisting of 52 points on the virtual hemisphere and one point at the origin (the large solid circle). There are four semicircles at azimuthal angular intervals of $45^{\circ}$, and each semicircle has 13 calculation points at $15^{\circ}$ intervals. The perpendicular direction to the surface of interest is assumed to be the incidence angle of $0^{\circ}$. Thus 52 intensities at the midpoints between all the points on the hemisphere and the center point were calculated. The sound intensity distribution as a function of the incidence angle at $15^{\circ}$ intervals for each frequency band can be com- puted by averaging the band-limited intensities from the same incidence angle, but different azimuth angles. After examining several receiver points, a spatially averaged intensity distribution on the surface of interest is obtained. The spatially averaged sound intensities on the surfaces of interest can be used as a weighting function for alleviating the discrepancy between the statistical and theoretical absorption coefficients, instead of adopting the uniform intensity assumption. Finally the corrected absorption coefficients were compared with the measured data in actual reverberation rooms in Sec. IV.

\section{Absorbing materials tested}

In this study, two porous materials with known surface impedance from Refs. 5 and 23 were tested, and the impedance data are listed in Tables I and II. Both of them are assumed to be locally reacting. Edge diffraction is not considered due primarily to lack of applicable edge diffraction analyses. Therefore it is presumed that the materials are mounted on the whole surface of interest in the test chambers.

The material from Ref. 23 is $5 \mathrm{~cm}$ thick rockwool with a density of $100 \mathrm{~kg} / \mathrm{m}^{3}$. The measured absorption coefficients quoted from the reference are the averaged absorption coefficients over 19 reverberation chambers. Three different surface areas of 4,8 , and $12 \mathrm{~m}^{2}$ were tested.

The second absorber from Ref. 5 is $5 \mathrm{~cm}$ thick and the specific flow resistance is $30 \mathrm{kNs} / \mathrm{m},{ }^{4}$ and the density is $50 \mathrm{~kg} / \mathrm{m}^{3}$. Three square specimens, the edges of which were $1.2,2.4$, and $3.6 \mathrm{~m}$, were measured in two reverberation chambers, and the volumes of which were 190 and $200 \mathrm{~m}^{3}$.

\section{SIMULATED SOUND INTENSITY DISTRIBUTIONS}

\section{A. Sound intensity distributions on the vertical wall of the irregular room}

Consider the porous material from Ref. 23 installed on the vertical surface of the irregular room illustrated in Fig.

TABLE II. Specific surface impedance data of the mineral wool from Ref. 5 .

\begin{tabular}{cccc}
\hline \hline $\begin{array}{c}\text { Center } \\
\text { frequency } \\
(\mathrm{Hz})\end{array}$ & $\begin{array}{c}\text { Specific } \\
\text { surface } \\
\text { impedance } \\
(\zeta)\end{array}$ & $\begin{array}{c}\text { Center } \\
\text { frequency } \\
(\mathrm{Hz})\end{array}$ & $\begin{array}{c}\text { Specific } \\
\text { surface } \\
\text { impedance } \\
(\zeta)\end{array}$ \\
\hline 100 & $1.0+10.1 j$ & 630 & $1.2+0.9 j$ \\
125 & $1.0+7.4 j$ & 800 & $1.3+0.7 j$ \\
160 & $1.0+5.5 j$ & 1000 & $1.4+0.6 j$ \\
200 & $1.1+4.3 j$ & 1250 & $1.5+0.4 j$ \\
250 & $1.1+3.3 j$ & 1600 & $1.6+0.4 j$ \\
315 & $1.1+2.5 j$ & 2000 & $1.4+0.3 j$ \\
400 & $1.1+1.8 j$ & 2500 & $1.2+0.3 j$ \\
500 & $1.1+1.3 j$ & 3150 & $1.2+0.3 j$ \\
\hline \hline
\end{tabular}



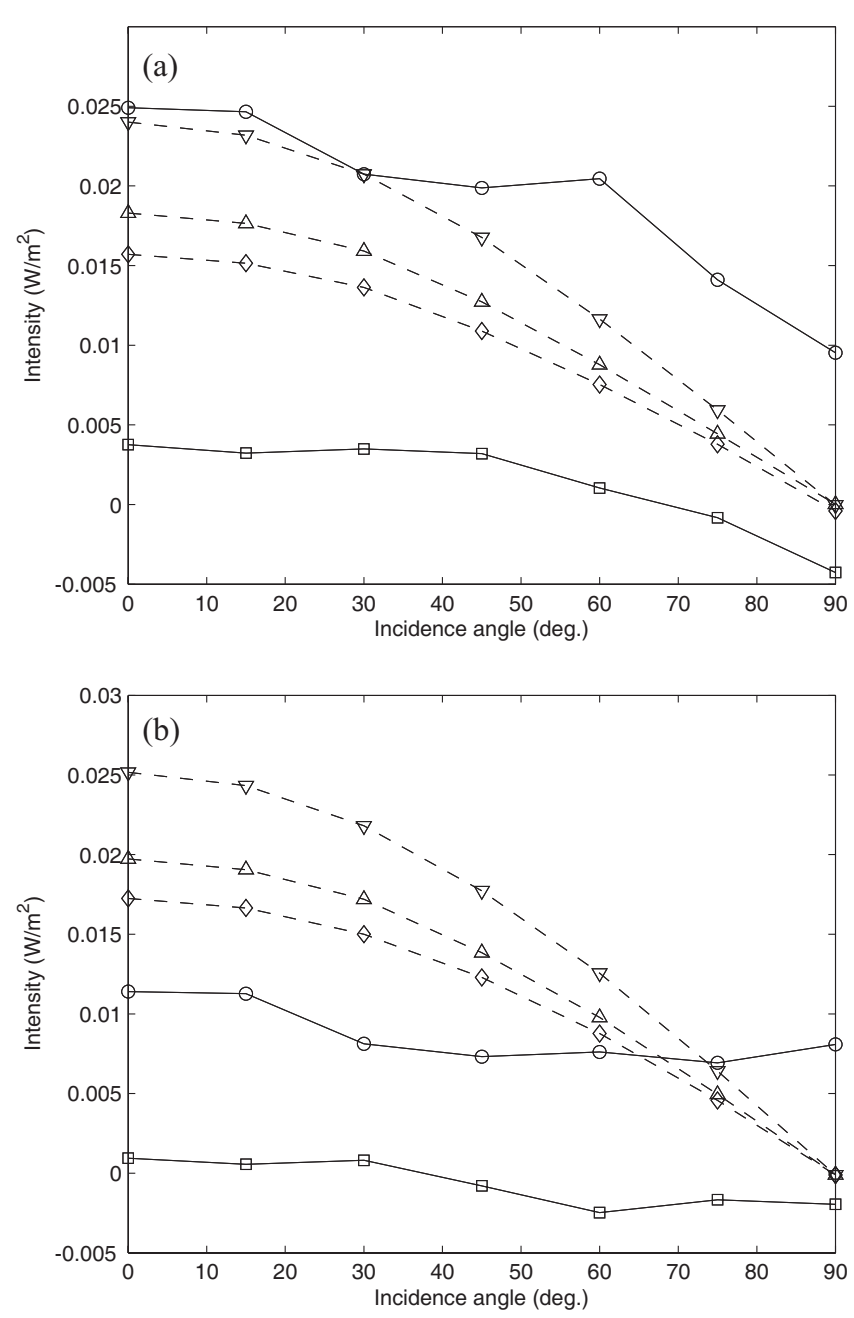

FIG. 3. Intensity distributions at a position $(2.7,0.001,2.5)$ : (a) use of $r_{\text {real }}$; (b) use of $r_{\text {comp. }}$ - $\bigcirc$ —: $250 \mathrm{~Hz} ;-\square —: 500 \mathrm{~Hz} ;--\diamond--: 1 \mathrm{kHz} ;--\triangle--: 2$ $\mathrm{kHz}$; and -- $\nabla--: 4 \mathrm{kHz}$.

2(a). When an omni-directional source is located at $(3.72,6$, 0.1 ), the sound intensity distribution at the central position of $(2.7,0.001,2.5)$ is shown in Fig. 3. It is noted that it is a sound intensity distribution at a single receiver point, not a spatially averaged distribution. The main difference in the sound intensity between the complex and real pressure reflection coefficients is highlighted in the lowest frequency band. The sound intensity distribution is flatter when using the complex pressure reflection coefficient. High frequency intensity distributions for both cases agree well, which fundamentally concurs with the postulation that wave interference is not important at high frequencies.

The spatially averaged sound intensity over the five reference points on the vertical surface is shown in Fig. 4. The spatially averaged sound intensity distribution is normalized to have the same area under the distribution with the uniform intensity distribution of unity. A difference in the intensity in the $250 \mathrm{~Hz}$ band is noticeable due to the consideration of the phase shifts on reflections. Except for the curve for the 250 $\mathrm{Hz}$ band, the other results overlap greatly in Figs. 4(a) and 4(b). The spatially averaged intensity distributions at higher frequencies agree well with a frequency-independent incident energy density calculated by the beam tracing method, ${ }^{17}$ which is displayed as the thick solid line.
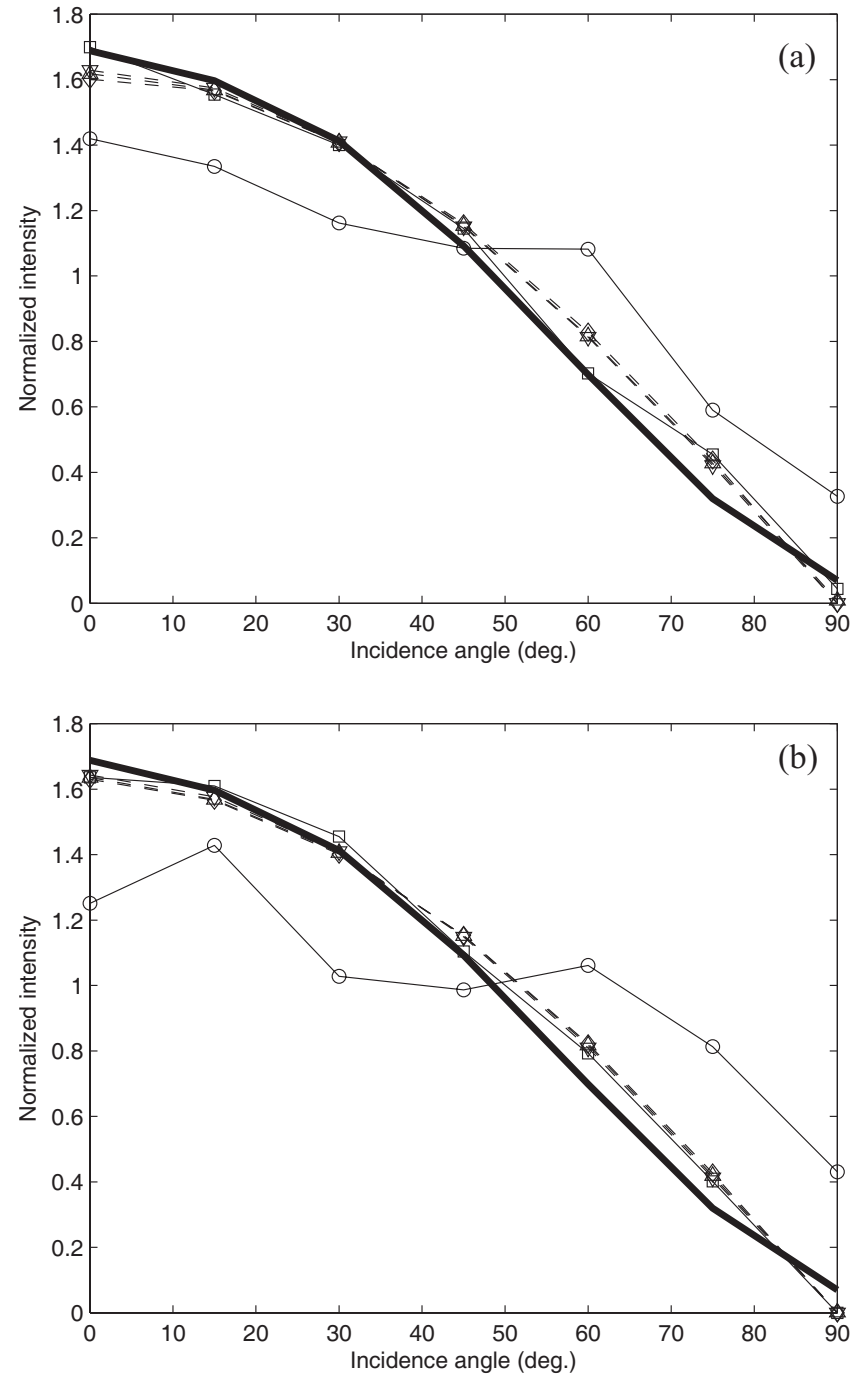

FIG. 4. Spatially averaged intensity distributions on the vertical wall in the irregular room. (a) use of $r_{\text {real }}$; (b) use of $r_{\text {comp. }}-\bigcirc —: 250 \mathrm{~Hz} ;-\square-: 500$ $\mathrm{Hz} ;--\diamond--: 1 \mathrm{kHz} ;--\triangle--: 2 \mathrm{kHz} ;--\nabla--: 4 \mathrm{kHz}$; and -: incident energy density (adapted from Ref. 17).

The sound intensity distribution on the absorber may be influenced by the absorption coefficient of the tested absorber. Two significantly different absorption coefficients of 0.2 and 1 were assigned to the vertical wall, and the results were compared in Fig. 5. When a totally absorbing specimen is installed on the vertical wall in the reverberation chamber, the sound field may be far from diffuse. The normally incident intensity is the strongest because of the shortest distance from the source to the wall, whereas the grazingly incident intensity is the weakest. Therefore the sound intensity decreases with the incidence angle. For such a condition, the intensity distributions are all similar to the Gaussian-like distribution shown in Fig. 4.

On the contrary, if the absorber has a fairly low absorption coefficient of 0.2 , the low frequency intensities start to deviate from the Gaussian-like distribution. The main difference from the total absorption case is a rather uniform intensity distribution for the lowest frequency band, the $250 \mathrm{~Hz}$ octave band. A possible reason for the more uniform distribution is the increased reverberant energy due to the acoustically hard surface. After the direct sound hits the vertical 

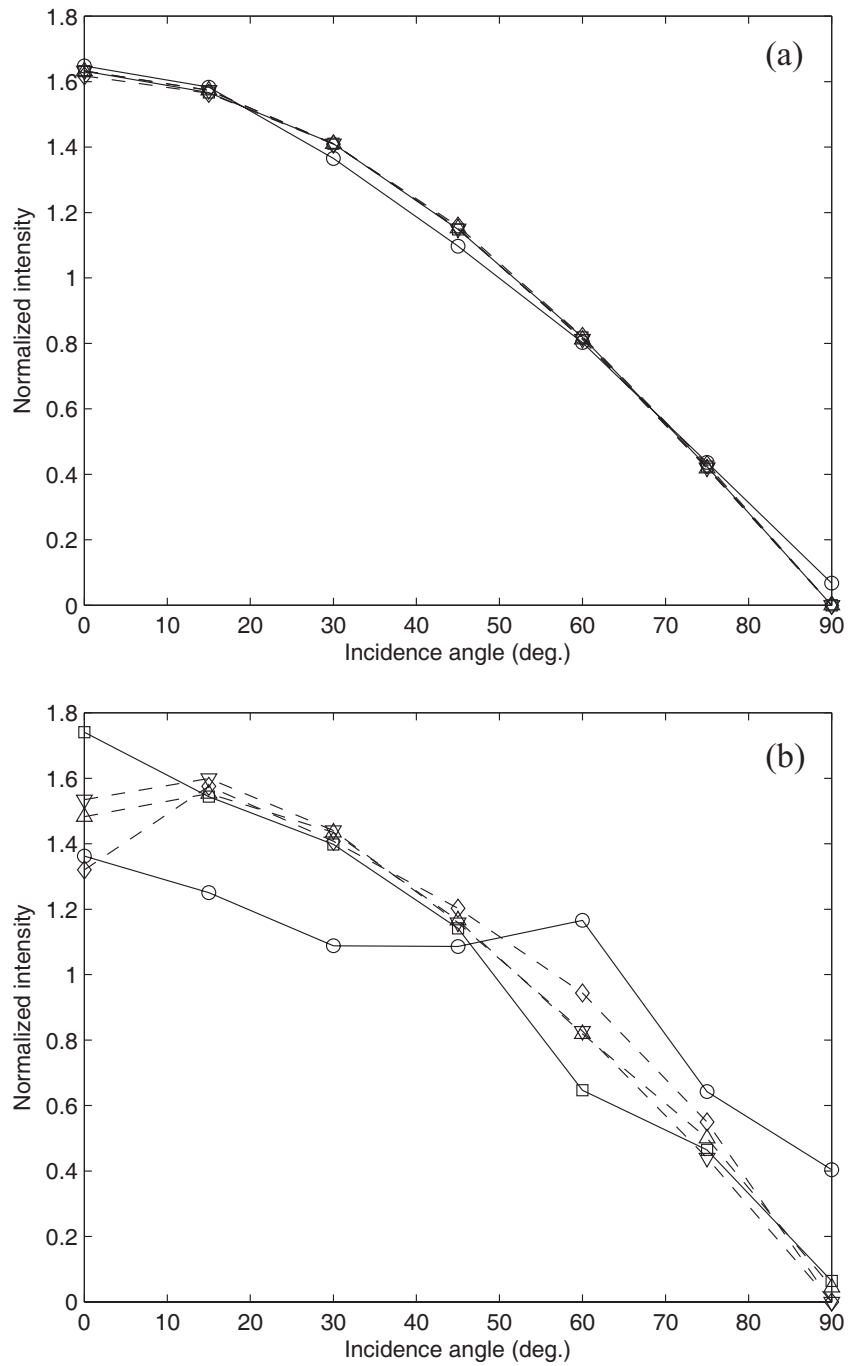

FIG. 5. Normalized intensity distributions on the absorber: (a) $\alpha_{s}=1$; (b) $\alpha_{s}=0.2$. —○—: $250 \mathrm{~Hz} ;-\square —: 500 \mathrm{~Hz} ;--\diamond--: 1 \mathrm{kHz} ;--\triangle--: 2 \mathrm{kHz}$; and $--\nabla--: 4 \mathrm{kHz}$

surface, sufficient energy rebounds into the sound field and lasts longer in the chamber, having higher chance of contributions from oblique angles. Therefore the intensity is redistributed, getting flatter at mid frequencies.

In both cases, the high frequency intensities above 1 $\mathrm{kHz}$ are all similar to one another, keeping the general tendency of decreasing intensity similar to the Gaussian-like distribution. This implies that the sound intensity distribution at high frequencies are likely to be a Gaussian-like distribution regardless of the absorption coefficient of the absorber.

\section{B. Sound intensity distributions on the floor of the irregular room}

Generally absorbers are installed on the floor of reverberation chambers when measuring absorption coefficients. If both the sound source and the receiver point are located close to the floor, the interference effect becomes more substantial, because the grazingly reflected component arrives nearly simultaneously with the direct sound at the receiver, which possibly results in destructive interference. Spatially averaged sound intensity distributions over five randomly
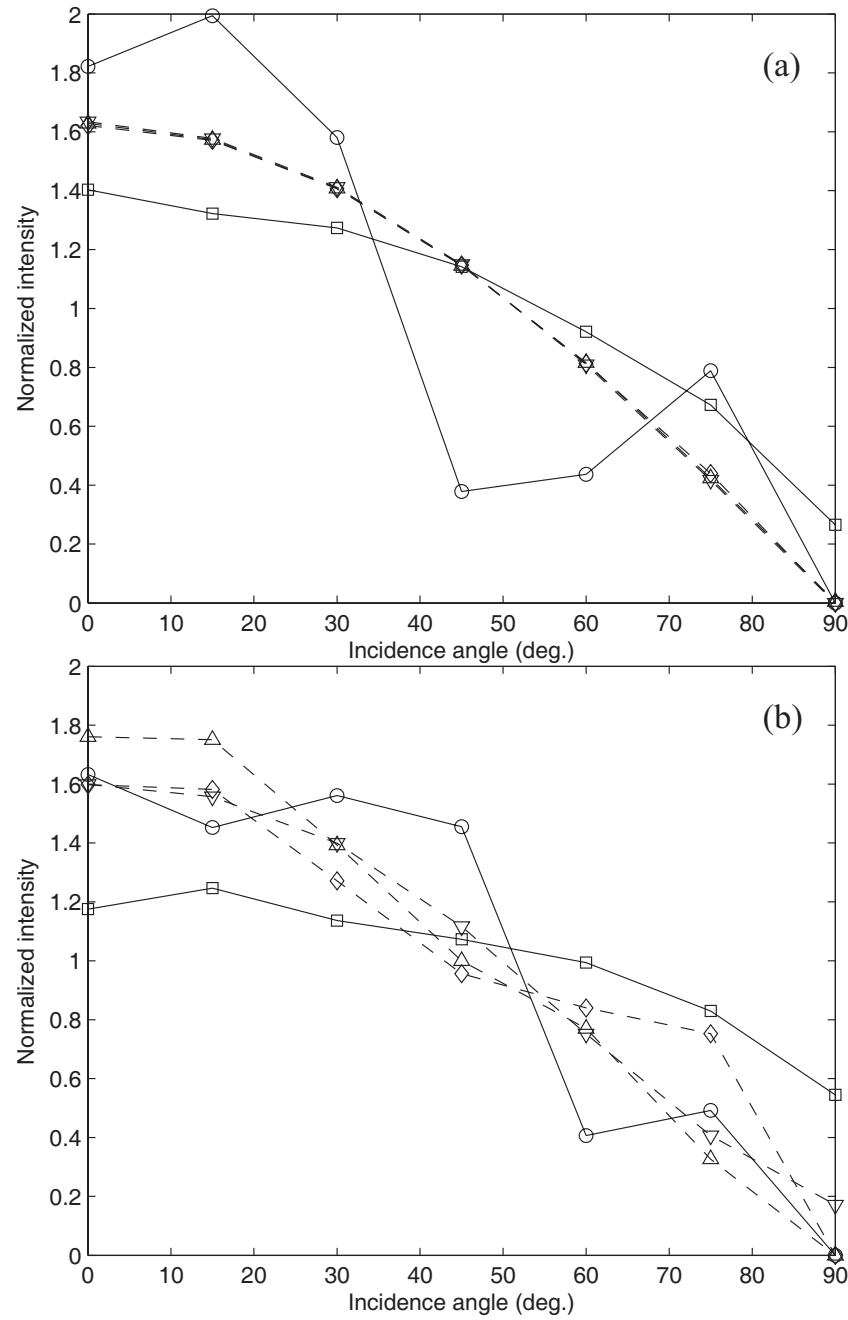

FIG. 6. Spatially averaged intensity distributions on the floor in the irregular room: (a) use of $r_{\text {real }}$; (b) use of $r_{\text {comp }}-\bigcirc —: 250 \mathrm{~Hz} ;-\square —: 500 \mathrm{~Hz}$; $--\diamond--: 1 \mathrm{kHz} ;--\triangle--: 2 \mathrm{kHz}$; and -- $\nabla--: 4 \mathrm{kHz}$.

chosen receivers employing two pressure reflection coefficients $r_{\text {real }}$ and $r_{\text {comp }}$ are compared in Fig. 6. Obviously the two results differ considerably due mainly to the interference effect.

\section{Sound intensity distributions on the floor of the rectangular room}

A spatially averaged sound intensity on the floor of the rectangular room employing the complex pressure reflection coefficient is shown in Fig. 7. Fairly uniform distributions for the 250 and $500 \mathrm{~Hz}$ bands, and Gaussian-like distributions for higher frequencies were obtained. Deviations between the intensities in the rectangular room and those in the irregular room in the 250 and $500 \mathrm{~Hz}$ bands can be explained by the different room geometries and the different absorber sizes.

It is noticed that the high frequency intensity distributions are always similar to the Gaussian-like distribution, as can be seen in Figs. 4-7. Since the high frequency intensity is nearly independent of the room geometry, the location of the absorber, and the absorption coefficient of the specimen, it can be generalized that the Gaussian-like distribution can 


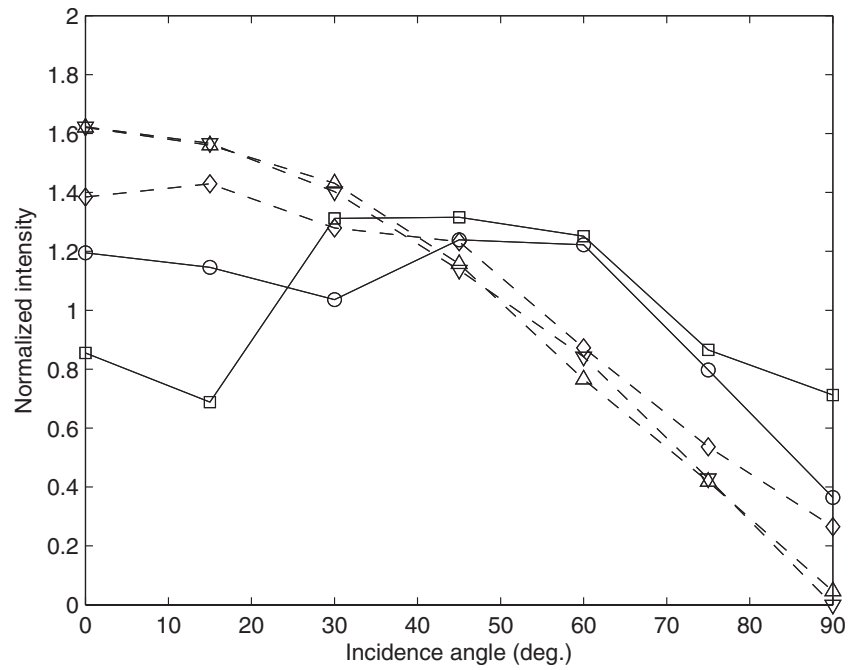

FIG. 7. Spatially averaged intensity distributions on the floor in the rectangular room employing $r_{\text {comp }}-\bigcirc-$ - $250 \mathrm{~Hz} ;-\square-: 500 \mathrm{~Hz}$; -- $\diamond--: 1$ $\mathrm{kHz} ;--\triangle--: 2 \mathrm{kHz}$; and -- $\nabla$--: $4 \mathrm{kHz}$.

be used for ordinary measurement conditions. The low frequency intensity distribution cannot be generalized; however, it is likely to be more uniform than the high frequency intensity distribution.

\section{APPLICATION OF WEIGHTING FUNCTIONS TO ABSORPTION COEFFICIENTS}

It is natural to believe that complex pressure reflection coefficients yield more accurate results than the real pressure reflection coefficient by taking account of interference of waves. Therefore the intensity distributions employing the complex pressure reflection coefficient have been utilized as weighting functions to correct the theoretical absorption coefficients. In addition to the non-uniform intensity correction, a size correction proposed by Thomasson ${ }^{27}$ was also employed. ${ }^{5}$ In his works, the absorption coefficient having a finite size is defined using the concept of the averaged radiation impedance as follows:

$$
\alpha_{\text {fin }}(\theta)=\frac{4 \operatorname{Re}\left(Z_{w}\right)}{\left|Z_{w}+\bar{Z}_{r}\right|^{2}} .
$$

Here, $Z_{w}$ is the surface impedance of the test specimen and $\bar{Z}_{r}$ is the averaged radiation impedance over azimuth angle from 0 to $2 \pi$ expressed as $\bar{Z}_{r}=(1 / 2 \pi) \int_{0}^{2 \pi} Z_{r} d \phi . Z_{r}$ is the radiation impedance, ${ }^{28}$ which is known to be $1 / \cos \theta$ for an infinitely large plate; however, the radiation impedance for a finite panel differs from $1 / \cos \theta$, in particular, near grazing incidence. $^{5,22,27}$ The radiation impedance for a finite panel is expressed as follows [see Eq. (A1) in Ref. 5 and Eq. (18) in Ref. 27]:

$$
Z_{r}=\frac{i k}{S} \iint_{S_{a}} \iint_{S_{a}} G e^{i k\left[\mu_{x}\left(x_{o}-x\right)+\mu_{y}\left(y_{o}-y\right)\right]} d x d y d x_{o} d y_{o},
$$

where $k$ is the wavenumber, $S=\iint_{S_{a}} d x d y, \mu_{x}=\sin \theta \cos \phi$, $\mu_{y}=\sin \theta \sin \phi, \quad G=-(2 \pi R)^{-1} \exp (i k R), \quad$ and $R$ $=\sqrt{\left(x-x_{o}\right)^{2}+\left(y-y_{o}\right)^{2}}$. Consequently an angle-and-size corrected absorption coefficient is calculated as follow:

$$
\begin{aligned}
\alpha_{\text {corrected }} & =2 \int_{0}^{\pi / 2} w(\theta) \cdot \alpha_{\text {fin }}(\theta) \cdot \sin (\theta) d \theta \\
& =2 \int_{0}^{\pi / 2} w(\theta) \cdot \frac{4 \operatorname{Re}\left(Z_{w}\right)}{\left|Z_{w}+\bar{Z}_{r}\right|^{2}} \cdot \sin (\theta) d \theta
\end{aligned}
$$

where $w(\theta)$ means the weighting function shown in Figs. 4(b), 6(b), and 7. Since the intensity distributions were computed in a limited frequency range from the $250 \mathrm{~Hz}$ to the 4 $\mathrm{kHz}$ octave band, it is assumed that intensity distributions below $250 \mathrm{~Hz}$ are considered the same as that in the $250 \mathrm{~Hz}$ octave band.

Figure 8 shows comparisons among measured statistical absorption, theoretical random incidence absorption by Eq. (1), size-corrected absorption using Eq. (6) and Paris' law, ${ }^{29}$ and three angle-and-size corrected absorption coefficients by Eq. (8). The consideration of the non-uniform intensity together with the size correction can compensate the overestimation of the measured statistical absorption coefficient. The size-corrected absorption coefficient seems to agree well with the measurement, but it is underestimated noticeably at high frequencies above $2 \mathrm{kHz}$.

As the size of the panel becomes larger, the angle-andsize corrected absorption coefficients agrees better with the measured data. If the absorber is installed on the vertical surface, which is not usual, a remarkable agreement is found except for the $500 \mathrm{~Hz}$ octave band. On the other hand, if the absorber is installed on the floor in the two rooms, the two angle-and-size corrected absorption coefficients agree well with the measured data for the entire frequency. The general trends of these two corrected absorption coefficients are similar, but the corrected absorption coefficient for the rectangular room is slightly higher. This might be ascribed to either the room volume effect as Kosten pointed out in Ref. 23 or the difference in the room volume and the absorbing area in the simulation of the intensity distribution.

Another noticeable point here is that the overall trend of the angle-and-size corrected absorption coefficient for the vertical installation of the absorber is similar to the random incidence absorption coefficient. They increase gradually until $1 \mathrm{kHz}$, whereas the measured data and the angle-and-size corrected absorption coefficients for the intensities on the floors show a gradual increase up to $500 \mathrm{~Hz}$ and become constant over the frequency. As discussed in Sec. I and Ref. 15 , a rather uniform angular distribution of incident energy is achieved by installing the absorber on the vertical surface, which eventually yields the overall trend similar to the random incidence absorption coefficient.

Relative errors were calculated with respect to the measured absorption coefficient as follows:

$$
\text { error }=\frac{1}{\mathrm{NB}} \sum_{n=1}^{\mathrm{NB}}\left|\frac{\alpha_{\mathrm{calc}}-\alpha_{\text {meas }}}{\alpha_{\text {meas }}}\right|,
$$

where $\alpha_{\text {meas }}$ is the measured statistical absorption coefficient, $\alpha_{\text {calc }}$ is the calculated absorption coefficients including the theoretical absorption, and NB is the number of frequency bands. In Table III, the average error of the angle-and-size corrected absorption coefficients is $6.5 \%$, showing a slight 


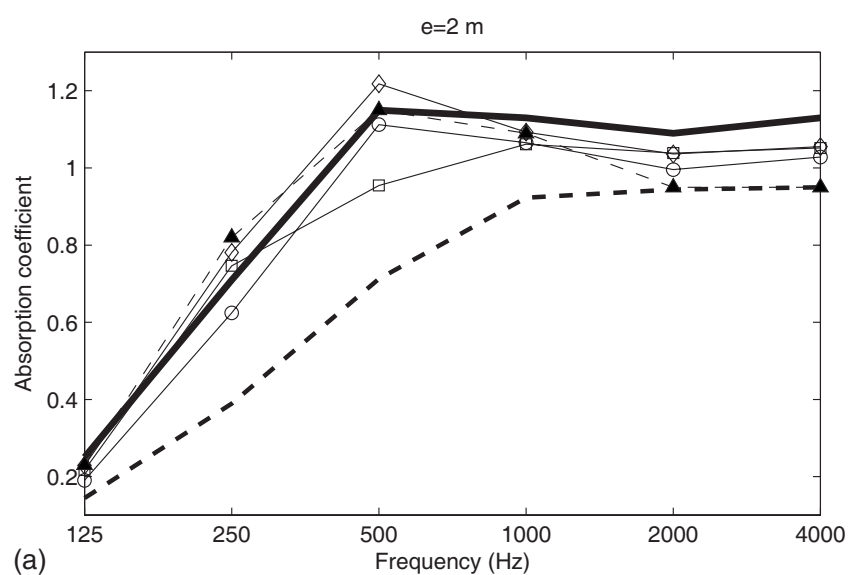

(a)
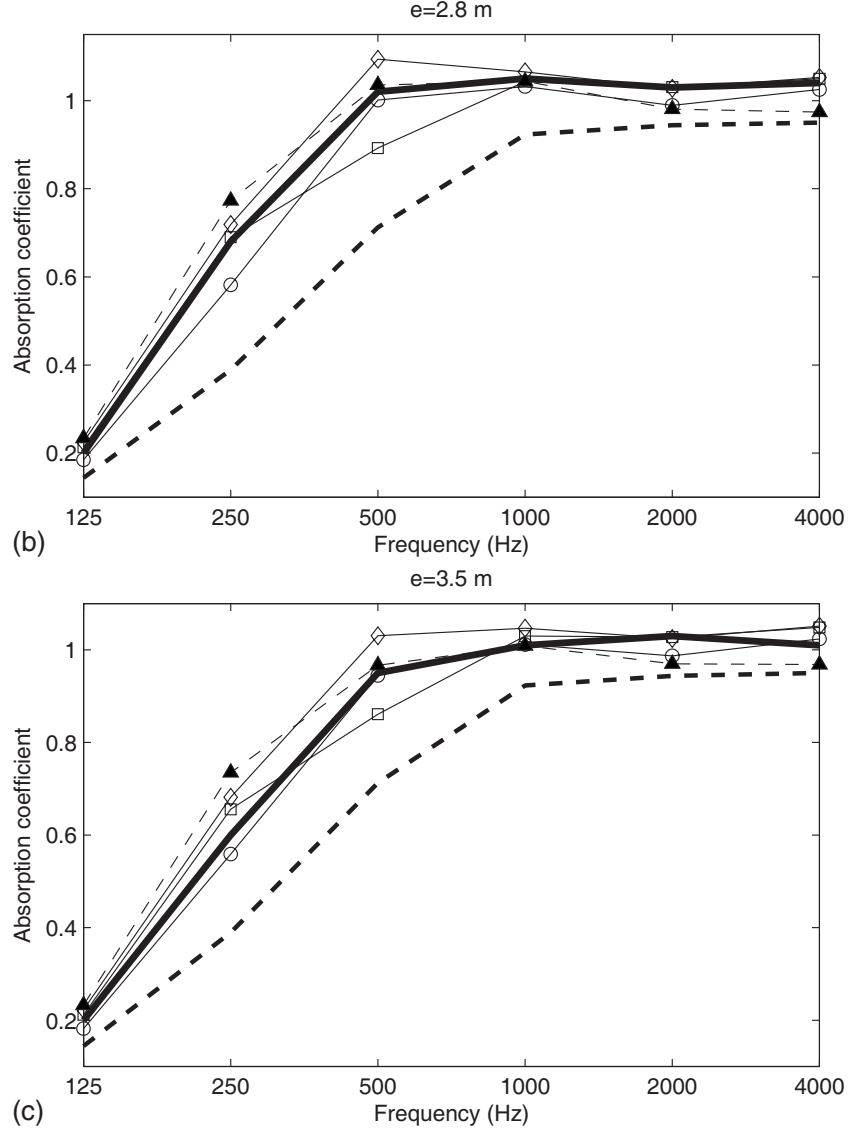

FIG. 8. Comparisons of measured and corrected absorption coefficients: (a) $S=4 \mathrm{~m}^{2}$, (b) $S=8 \mathrm{~m}^{2}$, and (c) $S=12 \mathrm{~m}^{2}$. — $\square$ : size-and-angle correction for the vertical wall of the irregular room; — $\bigcirc$ : size-and-angle correction for the floor of the irregular room; $-\diamond-$ : size-and-angle correction for the

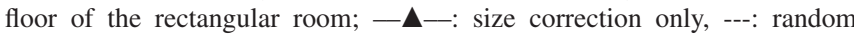
incidence; and -: measurement. improvement over that of the size-corrected one $(8.3 \%)$.

The absorber made of mineral wool from Ref. 5 was also tested using the simulated intensity distributions. Comparisons between the corrected absorption coefficients and the measurements are shown in Fig. 9 and Table III. Both the size-corrected and angle-and-size corrected absorption coefficients agree fairly well with the measurement, but the sizecorrected absorption coefficients start to underestimate at high frequencies.

Indeed, measured absorption coefficients depend largely on the room where the specimen is tested. Therefore it is regarded as best to simulate new weighting functions, particularly for low to mid frequencies, on a case-by-case basis. However the main challenge is that the sound intensity calculations necessitate the surface impedance data of the tested material, which are certainly not available for all materials to be tested. Since the high frequency intensity distributions do not vary significantly, one may use one of the calculated weightings in Figs. 4-7. At lower frequencies, the intensity distribution is likely to be more uniform, so a rather uniform distribution with decreasing intensity near grazing incidence can be assumed. It is finally emphasized that the nonuniform sound intensity is of primary importance at high frequencies, whereas the size effect may be more important at lower frequencies.

\section{CONCLUSIONS}

This study is concerned with an attempt to alleviate discrepancies between measured and theoretical absorption coefficients by considering actual non-uniform sound intensity distributions under test conditions. Sound intensity distributions in terms of the incidence angle on absorbers in two reverberation chambers have been simulated using a phased beam tracing, assuming that the absorbers completely cover two surfaces of interest. It is shown that the simulated intensity distributions actually vary with the room geometry, the absorption coefficient of the absorber, and the location of the absorber. The sound intensity at high frequencies decreases with the incidence angle, whereas it is rather uniform at low frequencies. For all studied cases, high frequency intensity distributions are similar and only small deviations are observed. The use of different pressure reflection coefficients leads to a noticeable change only in the low frequency intensity distributions. When the simulated intensity distributions

TABLE III. Relative errors of the calculated absorption coefficients normalized by the measured data.

\begin{tabular}{|c|c|c|c|c|c|}
\hline $\begin{array}{c}\text { Relative error } \\
(\%)\end{array}$ & $\begin{array}{l}\text { Random } \\
\text { incidence }\end{array}$ & $\begin{array}{c}\text { Size } \\
\text { corrected }\end{array}$ & $\begin{array}{c}\text { Size+ } \\
\text { non-uniform } \\
\text { (floor, irreg.) }\end{array}$ & $\begin{array}{c}\text { Size+ } \\
\text { non-uniform } \\
\text { (floor, rect.) }\end{array}$ & $\begin{array}{c}\text { Size+ } \\
\text { non-uniform } \\
\text { (vert., irreg.) }\end{array}$ \\
\hline Material $1(e=2 \mathrm{~m})$ & 28.8 & 9.3 & 10.4 & 6.5 & 8.8 \\
\hline Material $1(e=2.8 \mathrm{~m})$ & 21.6 & 7.3 & 5.1 & 4.7 & 3.7 \\
\hline Material $1(e=3.5 \mathrm{~m})$ & 18.5 & 8.4 & 3.6 & 6.9 & 5.1 \\
\hline Average error for the material from Ref. 23 & 23.9 & 8.3 & 6.4 & 6.0 & 5.9 \\
\hline Material $2(e=1.2 \mathrm{~m})$ & 32.3 & 6.0 & 3.7 & 4.2 & - \\
\hline Material $2(e=2.4 \mathrm{~m})$ & 24.6 & 4.5 & 5.0 & 4.4 & - \\
\hline Material $2(e=3.6 \mathrm{~m})$ & 21.6 & 5.6 & 5.7 & 5.4 & - \\
\hline Average error for the material from Ref. 5 & 26.2 & 5.4 & 4.8 & 4.7 & - \\
\hline
\end{tabular}



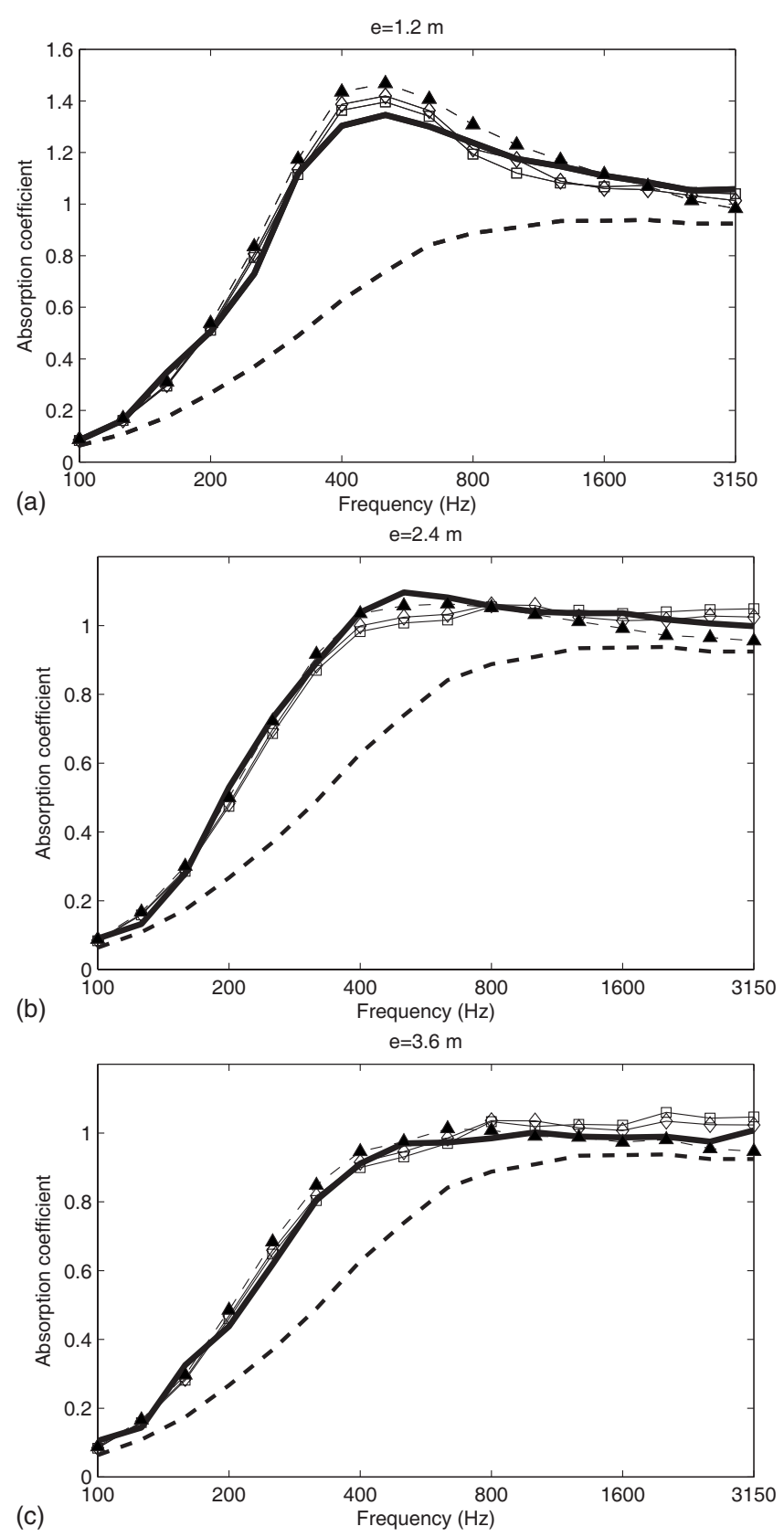

FIG. 9. Comparisons of measured and corrected absorption coefficient: (a) $e=1.2 \mathrm{~m}$, (b) $e=2.4 \mathrm{~m}^{2}$, and (c) $e=3.6 \mathrm{~m}$. - $\square$ - : size-and-angle correction for the floor of the irregular room; $-\diamond-$ : size-and-angle correction

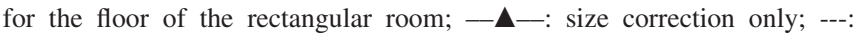
random incidence; and —: measurement.

were used as weighting functions, the corrected absorption coefficient agrees well with the measurement. The angleand-size corrected absorption coefficients agree best with the measurements, and a significant improvement over the sizecorrected absorption coefficient was achieved at high frequencies. Therefore it can be concluded that the non-uniform intensity distribution plays a key role in high frequency corrections, whereas the size correction is crucial at low frequencies.

\section{ACKNOWLEDGMENT}

The author is deeply indebted to a departed acoustician, Dr. Hyun-Ju Kang, for his initial suggestion of the study. The author also thanks Professor Finn Jacobsen for his help to improve the language and the clarity of this study.

${ }^{1}$ D. Olynyk and T. D. Northwood, "Comparison of reverberation room and impedance tube absorption measurement," J. Acoust. Soc. Am. 36, 21712174 (1964).

${ }^{2}$ H. Kuttruff, Room Acoustics, 4th ed. (Spon Press, London, 2000), pp. 46-49.

${ }^{3}$ R. K. Cook, "Absorption of sound by patches of absorbent material," J. Acoust. Soc. Am. 29, 324-329 (1957).

${ }^{4}$ A. D. Bruijn, "A mathematical analysis concerning the edge effect of sound absorbing materials," Acustica 28, 33-44 (1973).

${ }^{5}$ S.-I. Thomasson, "Theory and experiments on the sound absorption as function of the area," Report No. TRITA-TAK 8201, KTH, Stockholm (1982).

${ }^{6}$ A. C. C. Warnock, "The effect of room volume of measured sound absorption coefficient," in Proceedings of the 11th International Congress on Acoustics, pp. 3-6 (1983).

${ }^{7}$ P. Dammig, "Model investigation into sound fields in reverberation rooms," Acustica 75, 105-120 (1991).

${ }^{8} \mathrm{~V}$. L. Chrisler, "Dependence of sound absorption upon the area and distribution of the absorbent material," J. Res. Natl. Bur. Stand. 13, 169-187 (1934).

${ }^{9}$ R. E. Halliwell, "Inter-laboratory variability of sound absorption measurement," J. Acoust. Soc. Am. 73, 880-886 (1983).

${ }^{10} \mathrm{~T}$. W. Bartel, "Effect of absorber geometry on apparent absorption coefficients as measured in a reverberation chamber," J. Acoust. Soc. Am. 69, 1065-1074 (1981).

${ }^{11}$ T. D. Northwood and C. G. Balachandran, "Sound absorption coefficients and acoustical design," in Proceedings of the Third International Congress on Acoustics, pp. 847-849 (1961).

${ }^{12} \mathrm{M}$. C. Gomperts, "Do the classical reverberation formulae still have a right for existence?," Acustica 16, 256-268 (1965).

${ }^{13}$ P. M. Morse and R. H. Bolt, "Sound waves in rooms," Rev. Mod. Phys. 16, 69-150 (1944).

${ }^{14}$ F. V. Hunt, L. L. Beranek, and D. Y. Maa, "Analysis of sound decay in rectangular rooms," J. Acoust. Soc. Am. 11, 80-94 (1939).

${ }^{15}$ R. J. Donato, “Angular distribution of lower room modes," J. Acoust. Soc. Am. 41, 1496-1499 (1967).

${ }^{16}$ R. H. Bolt, "Normal modes of vibration in room acoustics: Angular distribution theory," J. Acoust. Soc. Am. 11, 74-79 (1939).

${ }^{17}$ C.-H. Jeong, "A correction of random incidence absorption coefficients for the angular distribution of acoustic energy under measurement conditions," J. Acoust. Soc. Am. 125, 2064-2071 (2009).

${ }^{18}$ C.-H. Jeong, J.-G. Ih, and J. H. Rindel, "An approximate treatment of reflection coefficient in the phased beam tracing method for the simulation of enclosed sound fields at medium frequencies," Appl. Acoust. 69, 601613 (2008).

${ }^{19}$ F. J. Fahy, Sound and Structural Vibration: Radiation, Transmission and Response (Academic Press, London, 1985), pp. 157-158.

${ }^{20}$ H.-J. Kang, J.-G. Ih, J.-S. Kim, and H.-S. Kim, "Prediction of sound transmission loss through multilayered panels by using Gaussian distribution of directional incident energy," J. Acoust. Soc. Am. 107, 1413-1420 (2000).

${ }^{21}$ Y. Makita and T. Hidaka, "Revision of the cosine theta law of oblique incident sound energy and modification of the fundamental formulations in geometrical acoustics in accordance with the revised law," Acustica 63, 163-173 (1987)

${ }^{22}$ J. H. Rindel, "Modeling the angle-dependent pressure reflection factor," Appl. Acoust. 38, 223-234 (1993).

${ }^{23} \mathrm{C}$. W. Kosten, "International comparison measurement in the reverberation room," Acustica 10, 400-411 (1960).

${ }^{24}$ M. Tohyama, H. Suzuki, and Y. Ando, The Nature and Technology of Acoustic Space (Academic Press, Tokyo, 1995), Chap. 4.1.

${ }^{25}$ ISO 354: Acoustics-Measurement of Sound Absorption in a Reverberation Room (International Organization for Standardization, Geneva, 2003).

${ }^{26}$ F. A. Everest, Master Handbook of Acoustics, 4th ed. (McGraw-Hill, New York, 2001), p. 324.

${ }^{27}$ S.-I. Thomasson, "On the absorption coefficient," Acustica 44, 265-273 (1980).

${ }^{28}$ P. F. E. Mechel, Formulas of acoustics (Springer, Berlin, 2002), pp. 214218.

${ }^{29}$ E. T. Paris, "On the coefficient of sound-absorption measured by the reverberation method," Philos. Mag. 5, 489-497 (1928). 